\title{
ON THE RELATION OF CORE MASS WITH CHEMICAL COMPOSITION IN PN
}

\author{
S.R. POTTASCH \\ Kapteyn Laboratory \\ P.O. Box 800 \\ 9700 AV Groningen \\ The Netherlands
}

\begin{abstract}
The attempts in the literature to find a relation between planetary nebula core mass and the nebular abundance ratios $\mathrm{N} / \mathrm{O}$ and $\mathrm{He} / \mathrm{H}$ are described. A critical discussion concludes that the evidence for such a relation is weak.
\end{abstract}

\section{Introduction}

It is clear that in the course of their evolution to planetary nebulae various elements have been produced in the nuclear burning stages, brought to the surface of the central star, and ejected so as to produce nebular overabundances. The evidence for this is quite direct. ${ }^{1}$ The oxygen abundance in PN is usually somewhat less than solar (see Fig. 1a), while the nitrogen to oxygen ratio varies from the solar value to an order of magnitude higher (see Fig. 1b). Since most stars must have begun their 'life' with approximately the solar value, it is inferred that the nitrogen was formed during the course of evolution of the central star. Similarly, helium and carbon are also found to have been enriched in the course of evolution of some, but not all, central stars. The other elements which have been well studied in PN, neon, and argon, are apparently not enriched. The helium and nitrogen enrichment are related to each other in a general way (see Fig. 2) and probably have been formed in the same or similar processes.

It is thought that the processes which lead to the enrichment of these elements are understood at least in a rudimentary way. These are discussed by Renzini and Voli (1981) and Iben and Renzini (1983) and many others (e.g. Groenewegen and De Jong, this volume). Three 'dredge-up' events are identified. The first occurs in all stars on the red giant branch following exhaustion of hydrogen in the core. Convection extending into the interior brings material processed in the $\mathrm{CN}$ cycle to the surface, resulting in an increase of nitrogen (by a factor of 2) and a decrease of both carbon (by $30 \%$ ) and the ${ }^{12} \mathrm{C} /{ }^{13} \mathrm{C}$ ratio. The second 'dredge-up' occurs only in higher mass stars (from about $2.3 \mathrm{M}_{\odot}$ to $8 \mathrm{M}_{\odot}$ ), and results in an increase of both He and $\mathrm{N}$ abundances. The third 'dredge-up' occurs along the AGB: Following each helium shell flash helium and carbon are convected to the surface. In these theories, oxygen is the least affected element and its abundance is expected to reflect that of the interstellar medium.

\footnotetext{
${ }^{1}$ Solar abundance is accepted as being similar to the abundance of the (interstellar) material out of which stars were formed.
} 


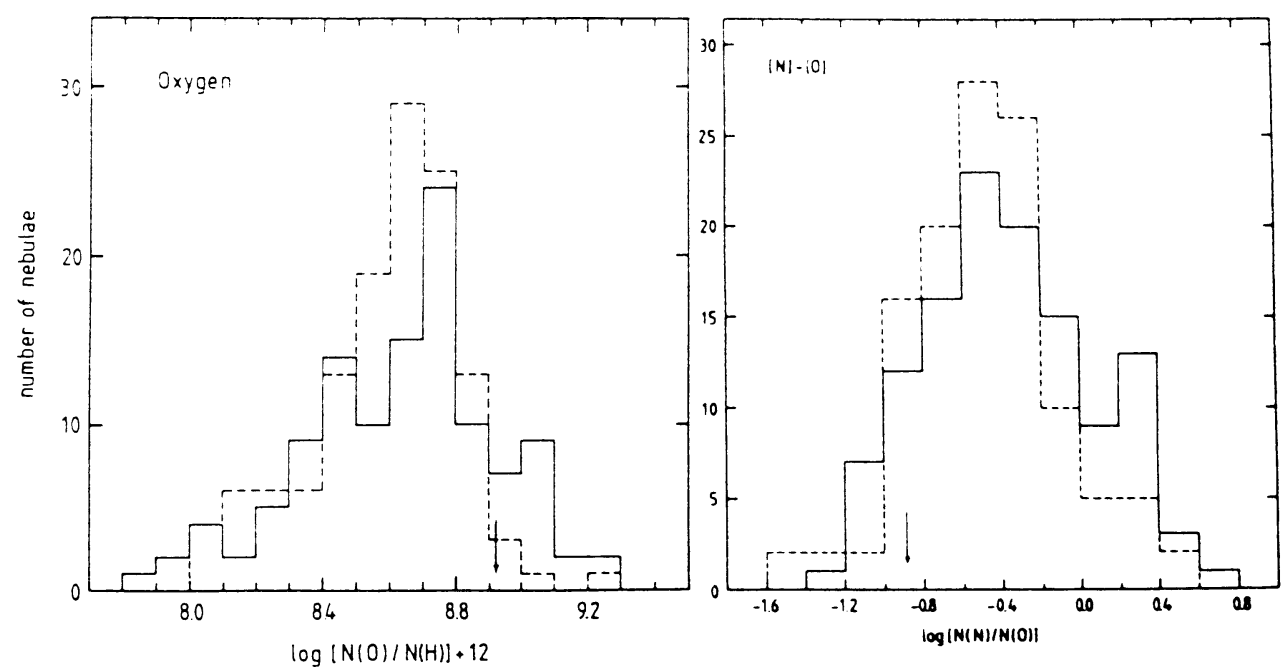

Fig. 1.

(a) Histogram of the oxygen abundance of galactic PN. Solid line bulge PN, dashed line 'nearby' PN. The arrow indicates the solar abundance. (b) same as (a) except that the nitrogen to oxygen ratio is varying.

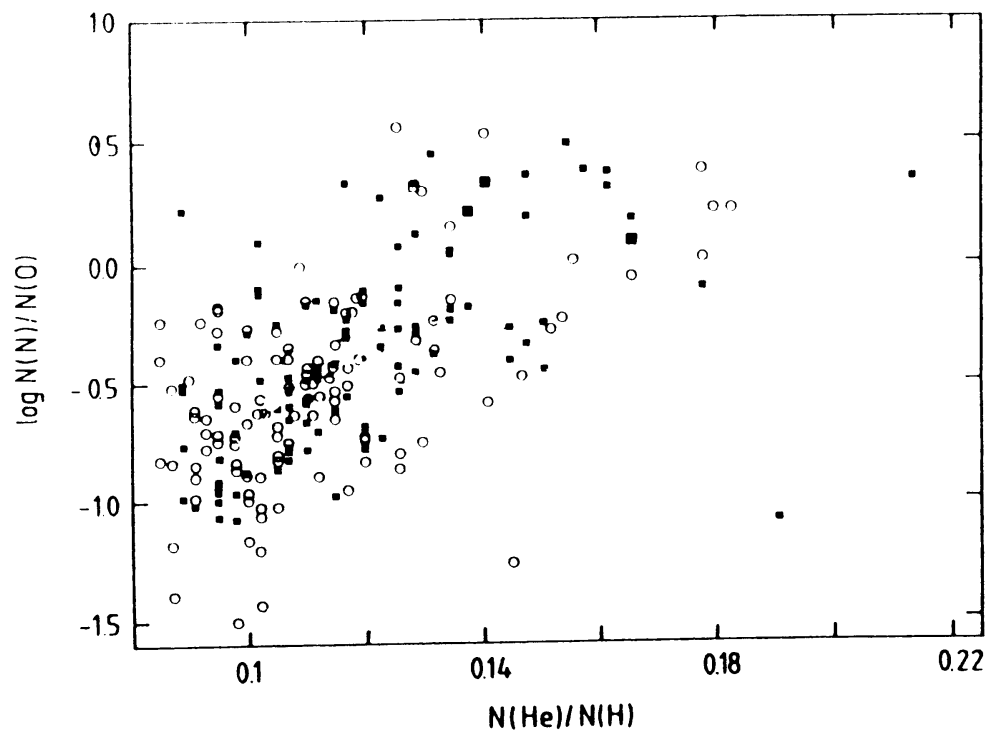

Fig. 2.

The nitrogen to oxygen ratio plotted against the $\mathrm{He} / \mathrm{H}$ ratio. The filled squares are the bulge PN, the open circles are the 'nearby' PN. 
There is a large uncertainty in the prediction of the enrichment. There are several reasons for this. The mixing length theory introduces parameters which are difficult to estimate. The amount of mass loss and its ejection are poorly known. Estimates of these quantities can be made which lead to predictions of the abundance ratio's $\mathrm{N} / \mathrm{O}, \mathrm{He} / \mathrm{H}$ and $\mathrm{C} / \mathrm{O}$ as functions of the initial mass of the star undergoing evolution. These predictions cannot be directly checked because the initial mass (or progenitor mass) that the central star had when on the main sequence cannot be found. One must therefore assume that there is a one-to-one relationship between the present central star mass (core mass) and the initial mass of the star.

The task of the present review is to summarize the present status as to whether there is an observed relationship between the core mass and the abundance ratios mentioned above. We will limit ourselves to the $\mathrm{N} / \mathrm{O}$ and $\mathrm{He} / \mathrm{H}$ ratio's because these are more widely observed and better known than the carbon abundance.

\section{Determining the Core Mass}

In a sense the determination of the core mass is straightforward. Paczynski (1971) demonstrated from models of stellar evolution that a relationship exists between the luminosity of the central star and its mass. The exact form of this relationship is somewhat uncertain. Besides the form given by Paczynski, forms are given by Schönberner (1981), Wood and Zarro (1981) and Boothroyd and Sackmann (1988) and others. For the present purpose it is not important which (if any) of these relations is the correct one. It is important that always the same relationship is used. This is not always the case in the literature so that caution is desirable.

The problem of determining the core mass is now shifted to the problem of determining the stellar luminosity. This is not so straightforward. First of all, there is the problem of determining the distance which for galactic PN is not easy to do accurately (see Terzian, this volume). Therefore a great deal of emphasis is placed of measurements of PN in the galactic bulge and the Magellanic Clouds, where distances are probably known to within $15 \%$. The luminosity can then be determined from measurements of the nebular lines if the nebula is optically deep in the ionizing radiation, i.e. all the stellar emission is absorbed by the nebula. There are various ways of estimating whether this is true or not (see Pottasch, 1993). We will not discuss this further, but use the estimates of the individual authors for the optical depth. If one has a doubt as to whether the nebula is optically deep, the luminosity can be determined from the stellar temperature and the observed visual magnitude assuming that the star radiates as a blackbody (or some known model atmosphere). This last procedure applies only to the galactic (bulge) nebulae, since it is usually very difficult (but not impossible) to see stellar radiation from the Magellanic Clouds.

Before the literature results of the luminosity vs abundance are given it is useful to try to obtain some insight as to what one would expect to see. 


\section{Some Insight}

What general results would one expect to see if:

1) the Schönberner (1981) tracks describe the evolution correctly

2) there is a monotonic relation between the core mass and the $\mathrm{N} / \mathrm{O}$ ratio.

In Fig. 3 the evolutionary tracks of Schönberner (1981) and Wood and Faulkner (1986) for various core masses have been plotted. The heavy lines indicate the ages of 3000 and 10000 years since the star had a surface temperature of $5000 \mathrm{~K}$. This is thought to be approximately when the last thermal pulse and expulsion of the nebula took place. Thus most of the observed PN will be expected to be found between the heavy lines or close to them.

Fig. 3.

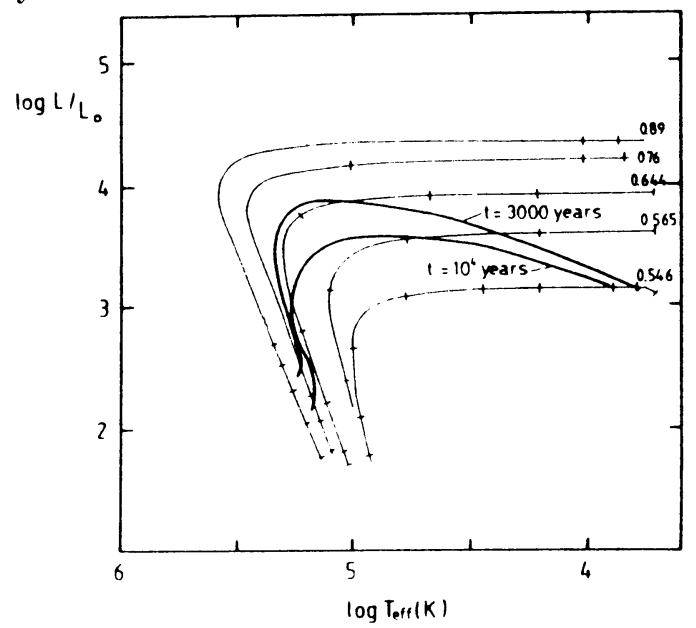

Theoretical evolutionary tracks (Schönberner, 1981; Wood and Faulkner, 1986). The thick lines indicate the ages of 3000 and 10000 years since the star had a surface temperature of $5000 \mathrm{~K}$.

Assume that the higher core mass stars have higher $\mathrm{N} / \mathrm{O}$ ratios. Then one would expect that:

(1) for central stars below $10^{5} \mathrm{~K}$ there will be a relation between central star temperature and $\mathrm{N} / \mathrm{O}$ ratio such that the higher temperatures have the higher $\mathrm{N} / \mathrm{O}$ ratio.

(2) that for central stars between $10^{5} \mathrm{~K}$ and $2 \times 10^{5} \mathrm{~K}$ (and with luminosities greater than $10^{2} \mathrm{~L}_{\odot}$ ) a wide range of $\mathrm{N} / \mathrm{O}$ will be found, but because those PN with higher $\mathrm{N} / \mathrm{O}$ will have central stars which evolved more quickly, these PN will have higher densities. One would therefore expect that for these nebulae there will be a relation between nebular density and $\mathrm{N} / \mathrm{O}$ ratio such that the higher densities correspond to higher $\mathrm{N} / \mathrm{O}$ ratio.

(3) PN with central stars having temperatures greater than $2 \times 10^{5} \mathrm{~K}$ will all have higher $\mathrm{N} / \mathrm{O}$ ratio's. 
Points (2) and (3) will be considered in the following section. Point (1) is illustrated in Fig. 4. The top figure is a plot of N/O against the central star temperature as taken from the work of Ratag et al (1991) for a large selection of galactic bulge $\mathrm{PN}$. The middle diagram is for a selection of 'nearby' $\mathrm{PN}$ where the $\mathrm{N} / \mathrm{O}$ ratio is taken from a variety of sources (Henry, 1990; de Freitas Pacheco et al, 1992; Perinotto, 1991; Stasinska and Tylenda, 1991) and the central star temperature is taken from a compilation of Zanstra temperatures by Zhang and Kwok (private communication, 1991). A diagram similar to this has been published by Stasinska and Tylenda (1991) for a somewhat different selection of galactic PN. The bottom diagram in the figure is for the LMC and is taken from the work of Dopita and Meatheringham (1991a,b). In this case other sources of data could have been used (e.g. Monk et al, 1988) but this was chosen because of the large numbers of PN measured in a uniform way. The temperature determination by Dopita and Meatheringham above $10^{5} \mathrm{~K}$ is questionable as will be discussed below.

Fig. 4.

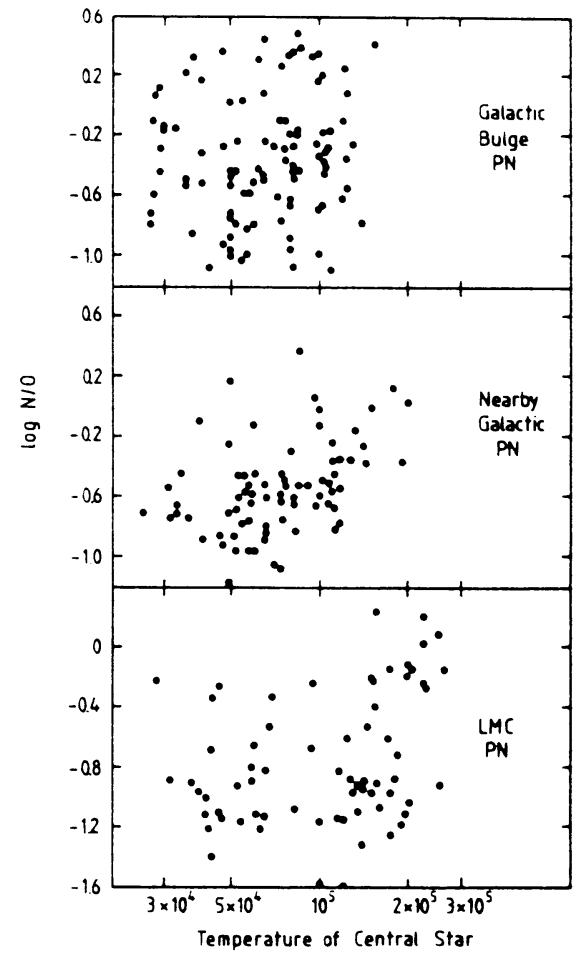

(a) N/O ratio plotted against central star temperature for galactic bulge PN (Ratag et al, 1991); (b) same as (a) but for a 'nearby' PN; (c) same as (a) but for LMC nebulae.

If only central stars with temperatures below $10^{5} \mathrm{~K}$ are considered, it appears 
very difficult to find any correlations in these diagrams. The diagrams for the galactic bulge and the LMC are clearly scatter diagrams, while any correlation in the diagram for the 'nearby' nebulae is at best marginal. This can also be said of the diagram of Stasinska and Tylenda (below $10^{5} \mathrm{~K}$ ). This negative result indicates that caution should be exercised in claims of correlations between $\mathrm{N} / \mathrm{O}$ and core mass.

\section{Results from the Literature}

The discussions in the literature are usually based on attempts to find a relation between the luminosity and the abundance ratios. We will discuss separately the LMC and the Galactic Bulge PN, where the distance in known, and the 'nearby' $\mathrm{PN}$, where the unknown distance is a further complication.

\subsection{PN IN THE LMC}

A large sample of LMC PN have been studied by Dopita and Meatheringham (1991a,b) using spectra taken by themselves, and by Kaler and Jacoby (1990, 1991) using spectra taken from several sources, especially Monk et al (1988) and the first paper by Dopita and Meatheringham. Both of these pairs of authors derive a temperature for the central star and a luminosity for each PN. A spectral criterion is given to distinguish between nebulae which are optically thick and thin in the Lyman continuum, and most are found to be optically thick.

The central star temperature derived in these studies are found from model fits to the spectrum assuming the star radiates as a blackbody. This is probably a reasonable approximation for temperatures less than $80,000 \mathrm{~K}$. For temperatures above $10^{5} \mathrm{~K}$, the temperature is essentially determined by the HeII $\lambda 4686$ to $\mathrm{H} \beta$ ratio. ${ }^{2}$ Very high stellar temperatures (sometimes above $2 \times 10^{5} \mathrm{~K}$ ) are obtained, which are probably unreliable for the following reasons:

1) Koppen and Preite-Martinez (1991) show that high ratios of $\lambda 4686 / \mathrm{H} \beta$ can be obtained even when the stellar temperature is as low as $50,000 \mathrm{~K}$.

2) If the method were applied to NGC 2392 a stellar temperature of almost $2 \times 10^{5} \mathrm{~K}$ is obtained while $50,000 \mathrm{~K}$ is the more accepted value. Likewise for NGC 4361 a value close to $3 \times 10^{5} \mathrm{~K}$ would be found, compared to the more accepted value of 80,000 to $100,000 \mathrm{~K}$ (Mendez et al, 1988; Torres-Peimbert et al, 1990).

The temperature thus found has no effect on the derived luminosities which depend mainly on the measured $\mathrm{H} \beta$ flux. It does strongly affect the core mass which is determined from the position on the HR diagram. These temperatures place the central star on the cooling curves of very high core mass models. This can be seen in Fig. 5 where the filled circles represent central stars of 'low temperatures' and the open circles are from the 'high temperature' central stars. While the former

\footnotetext{
${ }^{2} \mathrm{Kaler}$ and Jacoby use only this ratio. Dopita et al use the NeV line as well.
} 
do not have core masses higher than $0.64 \mathrm{M}_{\odot}$, the latter extend to as high as 1.2 $\mathrm{M}_{\odot}$.

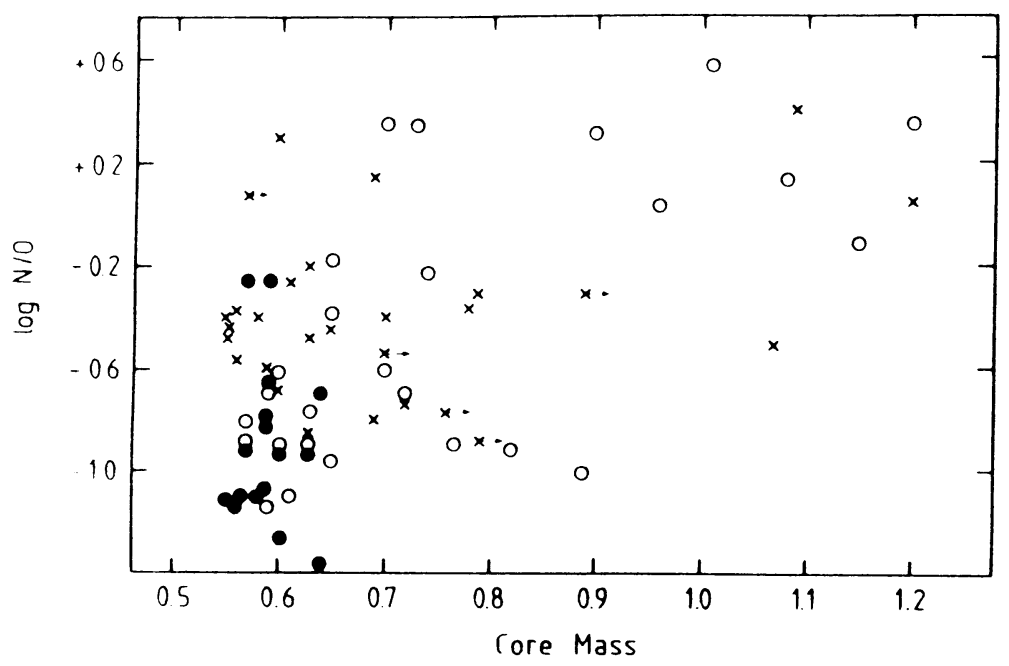

Fig. 5.

Core mass vs. nitrogen to oxygen ratio. The open circles are high temperature LMC central stars (Kaler and Jacoby, 1990) while the filled circles are LMC central stars with $\mathrm{T}<10^{5} \mathrm{~K}$ (Kaler and Jacoby, 1991). Results from Dopita and Meatheringham (1991a,b) are also included. The crosses are galactic central stars whose temperature is determined by the same method (Kaler et al, 1990).

Fig. 5 is the kind of plot presented by Kaler and Jacoby $(1990,1991)$ and Kaler et al (1990) as evidence for relation between core mass and nitrogen enrichment. There is clearly no correlation below core masses of $0.9 \mathrm{M}_{\odot}$. The correlation above $0.9 \mathrm{M}_{\odot}$ rests on the reliability of these high core masses and certainly needs further confirmation.

\subsection{PN IN THE GALACTIC BULGE}

A large sample of PN in the galactic bulge has been studied by Ratag et al (1991; 1993) using spectral measurements of Ratag et al (1992), Webster (1988) and Acker et al (1991). The central star temperatures are determined by a combination of the Energy Balance method, the Zanstra method and nebular models. The luminosity is then found by combining the stellar temperature and observed magnitude assuming a distance to the bulge of $7.8 \mathrm{kpc}$. The luminosity can also be determined from the $\mathrm{H} \beta$ flux for optically deep nebulae. Their resultant plot of luminosity against the nitrogen to oxygen ratio is shown as Fig. 6 . No correlation can be seen in this plot. The highest luminosity in Fig. 6 is less than $2 \times 10^{4} \mathrm{~L}_{\odot}$ which corresponds to a core mass of less than $0.75 \mathrm{M}_{\odot}$ Fig. 6 is therefore consistent with Fig. 5 which is also a scatter diagram below $0.75 \mathrm{M}_{\odot}$ But there appears no evidence from 
the Energy Balance or Zanstra methods for the high temperatures found from the HeII $\lambda 4686 / \mathrm{H}, 3$ ratio, and further brings into question the existence of core masses above $0.9 \mathrm{M}$.

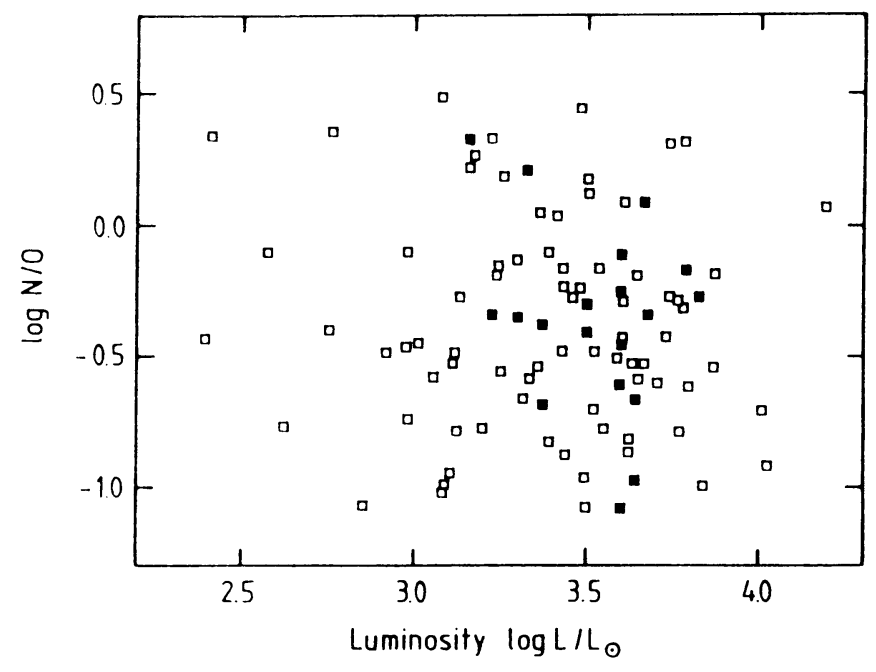

Fig. 6.

N/O ratio plotted against central star luminosity. Open squares are PN believed to be in 'horizontal' evolution; filled squares are PN already on the cooling track.

\subsection{NEARBY PLANETARY NEBULAE}

This is the most difficult group to discuss because the distances are most uncertain. Furthermore a selection effect enters because a limited group of PN are discussed at one time. Kaler et al (1990) select a group of large PN with high temperature central stars. They obtain distances from the Shklovski method. Central star temperatures are found essentially from the HeII $\lambda 4686 / \mathrm{H} \beta$ ratio, although sometimes the HII Zanstra temperature is considered. Because the method of determining the temperature is so similar to that of Kaler and Jacoby (1990), it is not surprising that the resultant core masses are also very similar. In fact, some of the results are also plotted in Fig. 5 .

Stasinska and Tylenda (1991) consider a sample with very little overlap with that of Kaler et al. Again distances are from the Shklovski method and central star temperatures are Zanstra determinations. Furthermore the theory plays an unclear role in their considerations, since they obtain three different core masses for a given nebula with a specified distance and central star temperature and luminosity. They find a possible correlation between the N/O ratio and core mass when one set of core masses is used. However when the core mass is determined from the position of the star on the HR diagram, the possible correlation disappears. 
One of the few PN common both to the discussion of Stasinska and Tylenda (1991) and Kaler et al (1990) is NGC 650. The former authors find a temperature of $107,000 \mathrm{~K}$ and a core mass of $0.59 \mathrm{M}_{\odot}$ for the central star, while the latter find a temperature of $175,000 \mathrm{~K}$ and a core mass greater than $0.89 \mathrm{M}_{\odot}$. This gross discrepancy is not due to the distance determination, which is the same in both cases.

Another argument against the high core masses derived by Kaler et al (1990) has been given by Napiwotzki and Schönberner (1991). Using high resolution spectra of the central star of PW 1 and a non LTE analysis, they derive a temperature $(60000-70000 \mathrm{~K})$, effective gravity $(\log \mathrm{g} \approx 7.5)$ and mass $\left(0.5\right.$ to $\left.0.6 \mathrm{M}_{\odot}\right)$ fot this object. This is quite different than given by Kaler et al, who assign a core mass of $1.0 \mathrm{M}_{\odot}$ to $\mathrm{PW} 1(\mathrm{~N} / \mathrm{O} \approx 1)$. Napiowotzki and Schönberner state that a hot white $\mathrm{d}$ warf of $1 \mathrm{M}_{\odot}$ is expected to have a $\log \mathrm{g} \approx 8.5$, which is in contradiction with the observed spectrum.

Gathier and Pottasch (1989) consider a sample of nebulae with independent distance determinations. They point out that there is a tendency that those with the highest luminosity also have the highest $\mathrm{N} / \mathrm{O}$ ratio. Their discussion is somewhat incomplete however since they make no distinction between high $\mathrm{He} / \mathrm{H}$ and high $\mathrm{N} / \mathrm{O}$.

The above approach, to consider individual objects, has advantages and disadvantages. The latter is that selection effects play an important role. For example the PN NGC 2440 and NGC 6537 have independent distance determinations. Their temperature is undoubtedly higher than $2 \times 10^{5} \mathrm{~K}$ (Heap et al, 1990). The luminosities suggest core masses of between 0.7 and $0.8 \mathrm{M}_{\odot}$. Both are known to have very high $\mathrm{N} / \mathrm{O}$ and $\mathrm{He} / \mathrm{H}$ ratios and it is tempting to cite these cases as evidence that high core mass objects have high $\mathrm{N} / \mathrm{O}$ ratios. The argument is even further strengthened since high stellar temperature is what is expected for high core mass objects. But counter examples exist: $2-3.3,3+3.1,351+5.1,355-4.2$. These are all galactic bulge objects with $\mathrm{N} / \mathrm{O}$ between 1 and 3 and high $\mathrm{He} / \mathrm{H}$ as well (in 3 cases). But they all have low temperature central stars and low to average luminosity (Ratag et al, 1993a). The nebulae are of average size. All of this indicates that they are low core mass objects having high $\mathrm{N} / \mathrm{O}$ and $\mathrm{He} / \mathrm{H}$ ratios.

It has also been suggested that NGC 2392 is a high core mass object, both from its high luminosity (Mendez et al, 1988) and from its suspected temperature increase in the past decade (Heap, 1992). But its $\mathrm{N} / \mathrm{O}$ ratio is about average and its $\mathrm{He} / \mathrm{H}$ ratio does not show a significant increase. Mention can also be made of the newly discovered PN, SAO 244567 (Parthasarthy et al, 1992), which has shown a strong increase in central star temperature over the past 40 years, yet it has only average $\mathrm{N} / \mathrm{O}$ and $\mathrm{He} / \mathrm{H}$ ratios. 


\section{Discussion}

The evidence that a relation exists between core mass and $\mathrm{N} / \mathrm{O}$ ratio is very weak. Fig. 7 is a histogram of all the available N/O ratios for PN in either the galactic bulge or in the LMC. In both groups distance uncertainties are unimportant. The central stars were divided in two temperature groups: Those below $75,000 \mathrm{~K}$ and those above $80,000 \mathrm{~K}$. Furthermore the low temperature group is subdivided into two luminosity categories: $\mathrm{L}<3000 \mathrm{~L}_{\odot}$ and $\mathrm{L} \geq 3000 \mathrm{~L}_{\odot}$. The values of temperature and luminosity are as given by the authors cited earlier.

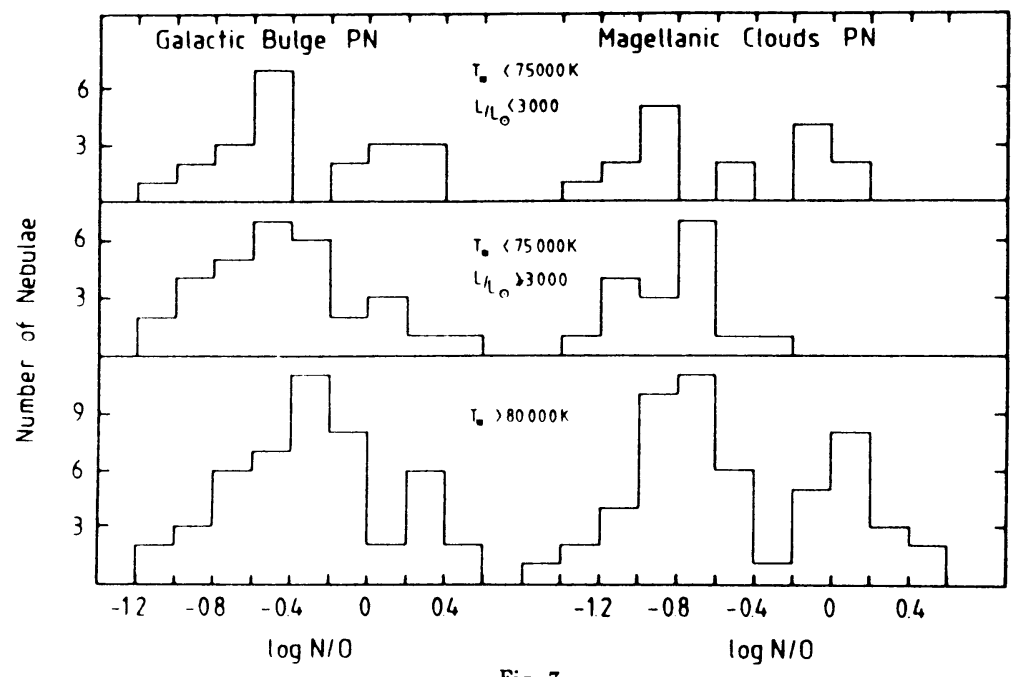

Fig. 7.

Histogram of the $\mathrm{N} / \mathrm{O}$ ratio in the Galactic Bulge and the LMC for various central star temperatures and luminosities.

For the low temperature objects, shown in the upper two diagrams in Fig. 7 both high and low values of $\mathrm{N} / \mathrm{O}$ are found. If the high core masses are found in the high luminosity group, as expected from the core mass-luminosity relation, we would expect to see a predominance of high $\mathrm{N} / \mathrm{O}$ ratios in the high luminosity group. This is definitely not seen, either in the LMC or in the galactic bulge objects.

In the lower diagram in Fig. 7 the high temperature objects are shown. While we have expressed doubts earlier about the values of the temperature found from the $\mathrm{HeII} \lambda 4686 / \mathrm{H} \beta$ ratio, it is still likely that all these objects have temperatures higher than $80,000 \mathrm{~K}$ and fall in this group. As can be seen from the figure, a wide range of $\mathrm{N} / \mathrm{O}$ is also found for this group. If there is a relation between core mass and $\mathrm{N} / \mathrm{O}$, those nebulae with high $\mathrm{N} / \mathrm{O}$ would be expected to have evolve much more quickly and therefore have higher densities than the $\mathrm{PN}$ with low N/O ratios (as discussed above). To see if this is true, the densities found from the [SII] or [OII] ratios have plotted in a histogram (Fig. 8) separately for those nebulae with high N/O ratios and those with low ratios. As can be seen, there is no indication 
in Fig. 8 that the PN with higher $\mathrm{N} / \mathrm{O}$ ratios also have higher densities, either in the galactic bulge or in the LMC.

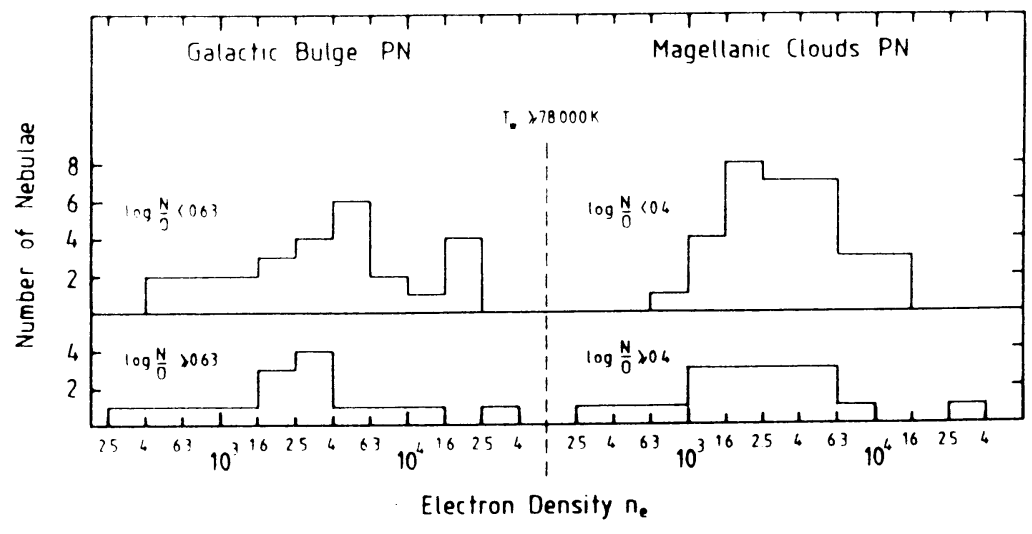

Fig. 8.

Histogram of the electron density for PN in the Galactic Bulge and the LMC. The $\mathrm{PN}$ are divided into groups with low (upper) and high $\mathrm{N} / \mathrm{O}$ ratio (lower).

This lack of evidence for a relation between $\mathrm{N} / \mathrm{O}$ and core mass makes it rather unlikely that a clearcut relation actually exists. It is possible that the luminosity has been incorrectly calculated, but this seems unlikely since it has been done by many groups independently. It is also unlikely that errors in the abundance ratio are large enough to cause such confusion.

It is possible that the assumption that the core mass is uniquely determined by the initial (progenitor) mass is wrong. Weidemann and Koster (1983), based on a study of white dwarfs in open clusters, show a trend that massive cores correspond to large initial masses. However, their relation shows a large scatter in the low mass range. It may be argued that this scatter is due to observational errors, but alternative explanations in terms of variation in mass loss efficiency have been advanced (Van der Veen and Habing, 1990).

Another possible source of confusion is the implicit assumption that hydrogen shell burning determines the evolutionary tracks. If there were a mixture of hydrogen and helium burning PN (which cannot at present be distinguished), an initial-final mass relation could exist, but we would be unable to see it.

\section{References}

Acker, A.A., Köppen, J., Stenholm, B., Raytchev, B. 1991, Astron. Astrophys. Suppl. 89, 237. Boothroyd, A.I., Sackmann, I.J. 1988, Astrophys. J. 328, 641.

Dopita, M.A., Meatheringham, S.J. 1991a, Astrophys. J. 367, 115.

Dopita, M.A., Meatheringham, S.J. 1991b, Astrophys. J. 377, 480.

de Freitas Pacheco, J.A., Maciel, W.J., Costa, R.D.D. 1992, Astron. Astrophys.. 
Gathier, R., Pottasch, S.R., 1989, Astron. Astrophys. 209, 369.

Groenewegen, M.A.T., De Jong, T. 1992, Astron. Astrophys. submitted.

Heap, S.R., Corcoran, M., Hintzen, P., Smith, E. 1990, in: From Mira to PN, ed. Mennessier M.-O. and Omont, A.

Henry, R.C. 1990, Astrophys. J. 356, 229; 363, 728.

Iben, I.J., Renzini, A. 1983, Ann. Rev. Astron. Astrophys. 21, 271.

Kaler, J.B., Jacoby, G.H. 1990, Astrophys. J. 362, 491.

Kaler, J.B., Jacoby, G.J. 1991, Astrophys. J. 382, 134.

Kaler, J.B., Shaw, R.A., Kwitter, K. 1990, Astrophys. J. 359, 392.

Köppen, J., Preite-Martinez, A. 1991, Astron. Astrophys. 248, 191.

Mendez, R.H., Kudritzki, R.P., Herrero, A., Husfeld, D., Groth, H.G. 1988, Astron. Astrophys. $190,113$.

Monk, D.J., Barlow, M.J., Clegg, R.E.S. 1988, Mon. Not. Roy. Astr. Soc. 234, 583.

Napiwotzki, R., Schönberner, D. 1991, in: White Dwarfs, ed. G. Vauclair, E. Sion, p. 39

Paczynski, B. 1971, Acta Astr. 21, 417.

Parthasarathy, M., Garcia-Lario, P., Pottasch, S.R., Manchado, A., Clavel, J., de Martino, D., v.d. Steene, G.S., Sahu, K.C. 1992 Astron. Astrophys..

Perrinoto, M. 1991, Astrophys. J. Suppl. Ser. 76, 687.

Pottasch, S.R. 1993, Astr. Astrophys. Rev.

Ratag, M.A. 1991, Thesis, Univ. of Groningen.

Ratag, M.A., Pottasch, S.R. Dennefeld, M., Menzies, J.W. 1992, Astron. Astrophys. Suppl. Ser.

Ratag, M.A., Pottasch, S.R. Dennefeld, M., Menzies, J.W. 1992, Astron. Astrophys.

Ratag, M.A., Pottasch, S.R., Water, L.B.F.M. 1993a, Astron. Astrophys.

Ratag, M.A., Waters, L.B.F.M., Pottasch, S.R. 1993b, Astron. Astrophys.

Renzini, A., Voli, M. 1981, Astron. Astrophys. 94, 175.

Schönberner, D. 1981, Astron. Astrophys. 103, 119.

Stasinska, G., Tylenda, R. 1990, Astron. Astrophys. 240, 467.

Torres-Peimbert, S., Peimbert, M., Pena, M. 1990, Astron. Astrophys. 233, 540.

V.d. Veen, W.E.C.J., Habing, H.J. 1990, Astron. Astrophys. 231, 404.

Webster, B.L. 1988, Mon. Not. Roy. Astr. Soc. 230, 377.

Weidemann, V., Koester, D., 1983 Astron. Astrophys. 121, 77.

Wood, P.R., Faulkner, D.J. 1986, Astrophys. J. 307, 659.

Wood, P.R., Zarro, D.M. 1981, Astrophys. J. 247, 247. 